Journal of Mathematics and Statistics 8 (2): 176-184, 2012

ISSN 1549-3644

(C) 2012 Science Publications

\title{
On the Successive Overrelaxation Method
}

\author{
I.K. Youssef \\ Department of Mathematics, \\ Faculty of Science, Ain Shams University, Cairo, 11 566, Egypt
}

\begin{abstract}
Problem statement: A new variant of the Successive Overrelaxation (SOR) method for solving linear algebraic systems, the KSOR method was introduced. The treatment depends on the assumption that the current component can be used simultaneously in the evaluation in addition to the use the most recent calculated components as in the SOR method. Approach: Using the hidden explicit characterization of linear functions to introduce a new version of the SOR, the KSOR method. Prove the convergence and the consistency analysis of the proposed method. Test the method through application to well-known examples. Results: The proposed method had the advantage of updating the first component in the first equation from the first step which affected all the subsequent calculations. It was proved that the KSOR can converge for all possible values of the relaxation parameter, $\omega^{*} \in \mathrm{R}-[-$ $2,0]$ not only for $(\omega \in(0,2)$ as in the SOR method. A new eigenvalue functional relation similar to that of the SOR method between the eigenvalues of the iteration matrices of the Jacobi and the KSOR methods was proved. Numerical examples illustrating this treatment, comparison with the SOR with optimal values of the relaxation parameter were considered. Conclusion: The relaxation parameter $\omega^{*}$ in the proposed method, can take values, $\omega^{*} \in \mathrm{R}-[-2,0]$ not only for $(\omega \in(0,2)$ as in the SOR. The enlargement of the domain has the affect of relaxing the sensivity near the optimum value of the relaxation parameter. Moreover, all the advantages of the SOR method are conserved and the proposed method can be applied to any system. This approach is promising and will help in the numerical treatment of boundary value problems. Other extensions and applications for further work are mentioned.
\end{abstract}

Key words: Linear systems, iterative methods, iteration matrices, successive over relaxation

\section{INTRODUCTION}

The problem of solving linear systems of algebraic equations appears as a final stage in solving many problems in different areas of science and engineering, it is the result of the discretization techniques of the mathematical models representing realistic problems (Saad and Vorst, 2000) and the references cited therein. We consider linear systems of the form Eq. 1:

$\sum_{j=1}^{m} a_{i j} x_{j}=b_{i}, i=1,2, \cdots, m$

We assume that the system has a unique solution and the equations are ordered so that, $\mathrm{a}_{\mathrm{ii}} \neq 0$, (Darvishi and Hessari, 2011; 2006; Papadomanolaki et al., 2010; Wang, 2010; Louka et al., 2009; Salkuyeh and Toutounian, 2006).

Jacobi method is the simplest known iterative method; it is a direct application of the fixed point theorem. The point Jacobi method for system (1) is Eq. 2:

$$
x_{i}^{[n+1]}=\frac{1}{a_{i i}}\left(b_{i}-\sum_{j=1}^{i-1} a_{i j} x_{j}^{[n]}-\sum_{j=i+1}^{m} a_{i j} x_{j}^{[n]}\right)
$$

From the computational point of view Gauss-Seidel method is a surprising natural extension for Jacobi method. Historically, Gauss introduced his method when he was working in least squares problem, in 1823, while Jacobi work appeared in 1853, (Saad and Vorst, 2000; Hackbusch, 1994). Gauss-Seidel idea depends on the use of the most recent calculated values. The point Gauss-Seidel method for system (1) is Eq. 3:

$$
\mathrm{x}_{\mathrm{i}}^{[\mathrm{n}+1]}=\frac{1}{\mathrm{a}_{\mathrm{ii}}}\left(\mathrm{b}_{\mathrm{i}}-\sum_{\mathrm{j}=1}^{\mathrm{i}-1} \mathrm{a}_{\mathrm{ij}} \mathrm{i}_{\mathrm{j}}^{[\mathrm{n}+1]}-\sum_{\mathrm{j}=\mathrm{i}+1}^{\mathrm{m}} \mathrm{a}_{\mathrm{ij}} \mathrm{x}_{\mathrm{j}}^{[\mathrm{n}]}\right)
$$

Moreover, the novel successive over relaxation approach, the SOR method, generalizes the Gauss Seidel method. The point SOR method for system (1) is Eq. 4 and 5:

$$
\left.\begin{array}{l}
x_{i}^{[n+1]}=x_{i}^{[n]}+\frac{\omega}{a_{i i}}(b_{i}-\underbrace{\sum_{j=1}^{i-1} a_{i j} x_{j}^{[n+1]}}_{\text {updated components }}-\underbrace{\sum_{j=i+1}^{m} a_{i j} x_{j]}^{[n]}-a_{i j} x_{j}^{[n]}}_{\text {old components }}) \\
\text { ond }
\end{array}\right)
$$


$\mathrm{x}_{\mathrm{i}}^{[\mathrm{n}+1]}=(1-\omega) \mathrm{x}_{\mathrm{i}}^{[\mathrm{n}]}+\omega \mathrm{x}_{\mathrm{GSi}}^{[\mathrm{n}+1]}$

where, $\omega \in(0,2)$ is a relaxation parameter, $\omega=1$ gives the Gauss Seidel method and $x_{\mathrm{Gsi}}^{[\mathrm{n}+1]}$ is the solution obtained by the Gauss Seidel method (Hackbusch, 1994; Burden and Faires, 2005; Varga, 1965).

Using matrix notations, the system of Eq. 1 can be written as Eq. 6:

$A X=b, X, b \in R^{m}, A \in R^{m \times m} A=D-L-U$

where, $\mathrm{D}$ is a diagonal matrix with the same diagonal elements as A and-L, - $U$ are the strictly lower and upper triangular parts of A respectively, (Hackbusch, 1994; Burden and Faires, 2005; Varga, 1965; Young, 1971).

Accordingly, we have.

\section{Jacobi method:}

$\mathrm{X}^{[\mathrm{n}+1]}=\mathrm{T}_{\mathrm{j}} \mathrm{X}^{[\mathrm{n}]}+\mathrm{D}^{-1} \mathrm{~b}, \mathrm{~T}_{\mathrm{j}}=\mathrm{D}^{-1}(\mathrm{~L}+\mathrm{U})$

$\mathrm{T}_{\mathrm{j}}$ is the Jacobi iteration matrix Eq. 7.

\section{Gauss-seidel method:}

$\mathrm{X}^{[\mathrm{n}+1]}=\mathrm{T}_{\mathrm{G}} \mathrm{X}^{[\mathrm{n}]}+(\mathrm{D}-\mathrm{L})^{-1} \mathrm{~b}, \mathrm{~T}_{\mathrm{G}}=(\mathrm{D}-\mathrm{L})^{-1} \mathrm{U}$

$\mathrm{T}_{\mathrm{G}}$ is the Gauss-Seidel iteration matrix Eq. 8.

\section{SOR method Eq. 9:}

$$
\begin{aligned}
& X^{[n+1]}=T_{S O R} X^{[n]}+(D-\omega L)^{-1} \omega b \\
& T_{\text {SOR }}=(D-\omega L)^{-1}((1-\omega) D+\omega U)
\end{aligned}
$$

$\mathrm{T}_{\mathrm{SOR}}$ is the SOR iteration matrix.

Definition: The spectral radius of a matrix $\mathrm{H}$, denoted $\rho(H)$, is given by:

$$
\rho(H)=\max \left\{\left|\lambda_{i}\right|: \lambda_{i} \text { is an eigen value of } H\right\}
$$

It is well known that a necessary and sufficient condition for the convergence of a given iterative method is that the spectral radius of the corresponding iteration matrix is less than one. The smaller the spectral radius of the iteration matrix is, the faster the rate of convergence of the corresponding iterative method is (Saad and Vorst, 2000; Hackbusch, 1994; Burden and Faires, 2005; Varga, 1965; Young, 1971; 1954).
The Gauss-Seidel and Successive Over-Relaxation (SOR) methods are important solvers for a class of large scale sparse linear systems due to their efficiency and simplicity in implementation. Many other surprising methods appeared in the last few decades used the same philosophy to introduce formulas that contain more parameters and include the other methods as special cases for some values of the parameters. The Accelerated Over Relaxation (AOR) method is a novel two parameter generalization of the above mentioned methods (Hadjidimos, 1978; Avdelas and Hadjidimos, 1981). Albrecht and Klein (1984), have considered extrapolated iterative methods, they have illustrated that the classical iterative methods can be interpreted as integration methods for certain systems of linear differential equations.

The basic idea in the KSOR method depends on the process of updating the residue in the right hand side of the SOR method (4). It is assumed that the current value can be used in addition to the use of the most recent calculated ones (i.e., updating the residue simultaneously with the current new component). Apparently, this process leads to an implicit formula but actually it is explicit due to the linearity of the equations. Accordingly, the first component is updated in the first step which affects all the subsequent steps. Unlike Gauss-Seidel (SOR), AOR and the extrapolated versions of iterative methods in which the solution is updated after the determination of the new component in the KSOR it is assumed that the update prosses can take place simultaneosoully with the evaluation of the new components. The iteration matrix of the proposed method is obtained, theoretical considerations are being discussed. It is proved that the method is completely consistent and can converge for values of the relaxation parameter $\left(\omega^{*} \in \mathrm{R}-[-2,0]\right)$ not only for the relaxation parameter $(\omega \in(0,2))$ in the SOR. Moreover, the proposed method will be convergent when the classical SOR $(\omega \in(0,2))$ is convergent. Comparison of the results of the proposed method with other well- known iterative methods especially with SOR with optimal values of the relaxation parameter $\omega$ has proved the efficiency and reliability of the method. Numerical examples with the graphical behavior of the spectral radius of the corresponding iteration matrices as a function in $\omega^{*}$ are discussed. Moreover, the proposed KSOR method has the same simple explicit appearance as the SOR method.

\section{MATERIALS AND METHODS}

Assuming that we can use the current component simultaneously on the evaluation of the residue appears in the SOR method in addition to the use of the most 
recent calculated one. It appears that the method will be implicit; however after the rearrangement of the terms, we get an explicit formula. Accordingly, the KSOR method can be written in the form Eq. 10-12:

$$
x_{i}^{[n+1]}=x_{i}^{[n]}+\frac{\omega^{*}}{a_{i i}}(b_{i}-\underbrace{\sum_{j=1}^{i-1} a_{i j} x_{j}^{[n+1]}}_{\text {updated }}-\underbrace{\sum_{j=1+1}^{m} a_{i j} x_{j}[n]}_{\text {old }}-\underbrace{a_{i j} x_{j}^{[n+1]}}_{\substack{\text { Aspumed } \\ \text { updated }}})
$$

$\mathrm{i}=1,2, \ldots, \mathrm{m}, \omega \omega^{*} \mathrm{i} \mathrm{R}-[-2,0]$

$$
\begin{aligned}
& x_{i}^{[n+1]}=\frac{1}{\left(1+\omega^{*}\right)} \times\left\{x_{i}^{[n]}+\frac{\omega^{*}}{a_{i i}}\left(b_{i}-\sum_{j=1}^{i-1} a_{i j} x_{j}^{[n+1]}-\sum_{j=i+1}^{m} a_{i j} X_{j}^{[n]}\right)\right\} \\
& i=1,2, \cdots, m, \omega^{*} \in R-[-2,0]
\end{aligned}
$$$$
\mathrm{x}_{\mathrm{i}}^{[\mathrm{n}+1]}=\frac{1}{\left(1+\omega^{*}\right)} \mathrm{x}_{\mathrm{i}}^{[\mathrm{n}]}+\frac{\omega^{*}}{\left(1+\omega^{*}\right)} \mathrm{x}_{\mathrm{GSi}}^{[\mathrm{n}+1]}
$$

$\mathrm{i}=1,2, \cdots, \mathrm{m}, \omega^{*} \in \mathrm{R}-[-2,0]$

The relaxation parameter $\omega^{*} \in \mathrm{R}-[-2,0]$ plays the same role as $\omega$ in the SOR method but with extended domain. It is used to control the spectral radius of the iteration matrix, accordingly the rate of convergence.

The matrix formulation of the KSOR method is Eq. 13 and 14 :

$$
\begin{aligned}
& \mathrm{X}^{[\mathrm{n}+1]}=\mathrm{T}_{\mathrm{KSOR}} \mathrm{X}^{[\mathrm{n}]}+\left(\left(1+\omega^{*}\right) \mathrm{D}-\omega^{*} \mathrm{~L}\right)^{-1}\left(\omega^{*} \mathrm{~b}\right) \\
& \mathrm{T}_{\mathrm{KSOR}}=\left(\left(1+\omega^{*}\right) \mathrm{D}-\omega^{*} \mathrm{~L}\right)^{-1}\left(\mathrm{D}+\omega^{*} \mathrm{U}\right)
\end{aligned}
$$

where, $\mathrm{T}_{\mathrm{KSOR}}$ is the iteration matrix of the KSOR method.

We first prove a basic result which gives the maximum range of values of $\omega^{*}$ for which the KSOR iteration can converge.

Theorem 1: Let $A \in \mathrm{R}^{\mathrm{m} \times \mathrm{m}}$ with $\mathrm{a}_{\mathrm{ii}} \neq 0$. Then $\rho\left(\mathrm{T}_{\mathrm{kSOR}}\right) \geq \frac{1}{\left|1+\omega^{*}\right|}$, which implies that the KSOR method can converge for all $\omega^{*} \in \mathrm{R}-[-2,0]$

Proof: for all, $\omega^{*} \neq-1$, we have:

$$
\begin{aligned}
& \operatorname{det}\left(\mathrm{T}_{\mathrm{KSOR}}\right)=\operatorname{det}\left(\left(\left(1+\omega^{*}\right) \mathrm{D}-\omega^{*} \mathrm{~L}\right)^{-1}\left(\mathrm{D}+\omega^{*} \mathrm{U}\right)\right) \\
& =\operatorname{det}\left(\left(1+\omega^{*}\right) \mathrm{D}-\omega^{*} \mathrm{~L}\right)^{-1} \operatorname{det}\left(\mathrm{D}+\omega^{*} \mathrm{U}\right) \\
& \operatorname{det}\left(\mathrm{T}_{\mathrm{KSOR}}\right)=\frac{1}{\operatorname{det}\left(\left(1+\omega^{*}\right) \mathrm{D}-\omega^{*} \mathrm{~L}\right)} \operatorname{det}\left(\mathrm{D}+\omega^{*} \mathrm{U}\right)
\end{aligned}
$$

$$
\begin{aligned}
& =\frac{1}{\operatorname{det}\left(\left(1+\omega^{*}\right) \mathrm{D}\right)} \operatorname{det}\left(\mathrm{D}+\omega^{*} \mathrm{U}\right) \\
& =\frac{1}{\left(1+\omega^{*}\right)^{\mathrm{m}} \operatorname{det}(\mathrm{D})} \operatorname{det}\left(\mathrm{D}+\omega^{*} \mathrm{U}\right) \\
& =\frac{1}{\left(1+\omega^{*}\right)^{\mathrm{m}}} \operatorname{det}(\mathrm{D})^{-1} \operatorname{det}\left(\mathrm{D}+\omega^{*} \mathrm{U}\right) \\
& \operatorname{det}\left(\mathrm{T}_{\text {KSOR }}\right)=\frac{1}{\left(1+\omega^{*}\right)^{\mathrm{m}}} \operatorname{det}\left(\mathrm{I}+\omega^{*} \mathrm{D}^{-1} \mathrm{U}\right)=\frac{1}{\left(1+\omega^{*}\right)^{\mathrm{m}}}
\end{aligned}
$$

Since, $\operatorname{det}\left(\mathrm{T}_{\mathrm{KSOR}}\right)=\prod_{\mathrm{j}=1}^{\mathrm{m}} \beta \mathrm{j}$

where, $\beta_{\mathrm{j}}$ is the eigenvalues of the iteration matrix, $\mathrm{T}_{\mathrm{KSOR}}$, accordingly we find:

$$
\left|\prod_{j=1}^{m} \beta_{j}\right|=\left|\operatorname{det}\left(T_{\text {KSOR }}\right)\right|=\left|\frac{1}{\left(1+\omega^{*}\right)^{m}}\right| \leq \max \left|\beta_{j}\right|^{m}
$$

Thus $\rho\left(\mathrm{T}_{\mathrm{KSOR}}\right) \geq \frac{1}{\left|1+\omega^{*}\right|}$. For convergence, we must have $1>\rho\left(\mathrm{T}_{\mathrm{KSOR}}\right) \geq \frac{1}{\left|1+\omega^{*}\right|}$ and this gives $\omega^{*} \in \mathrm{R}-[-2,0]$.

Theorem 2: The KSOR method (10 or 11) is completely consistent with the system (1) for all values of the relaxation parameter $\omega^{*} \in \mathrm{R}-[-2,0]$.

Proof: The proof is straightforward application of the definition of consistency (Young, 1971).

Theorem 3: The characteristic equation of the KSOR iteration matrix can be written in the form Eq. 15:

$\operatorname{det}\left(\left(1-\beta-\beta \omega^{*}\right) D+\omega^{*} U+\omega^{*} \beta L\right)=0, \omega^{*} \notin[-2,0]$

Proof: the characteristic equation is:

$$
\begin{aligned}
& \operatorname{det}\left(\mathrm{T}_{\text {KSOR }}-\beta \mathrm{I}\right)=0 \\
& \operatorname{det}\left[\left(\left(1+\omega^{*}\right) \mathrm{D}-\omega^{*} \mathrm{~L}\right)^{-1}\left(\mathrm{D}+\omega^{*} \mathrm{U}\right)-\beta \mathrm{I}\right]=0 \\
& \operatorname{det}\left(\left(1+\omega^{*}\right) \mathrm{D}-\omega^{*} \mathrm{~L}\right)^{-1} \operatorname{det}\left[\begin{array}{l}
\left(\mathrm{D}+\omega^{*} \mathrm{U}\right)-\beta \times \\
\left(\left(1+\omega^{*}\right) \mathrm{D}-\omega^{*} \mathrm{~L}\right)
\end{array}\right]=0 \\
& \text { Since } \operatorname{det}\left(\left(1+\omega^{*}\right) \mathrm{D}-\omega^{*} \mathrm{~L}\right) \neq 0
\end{aligned}
$$

We must have:

$$
\operatorname{Det}\left[\left(1-\beta-\beta \omega^{*}\right) D+\omega^{*} U+\omega^{*} \beta L\right]=0
$$


This result holds for any system of the form (1) (have a unique solution with $\mathrm{a}_{\mathrm{ii}} \neq 0$. Moreover, for any $\omega^{*} \in \mathrm{R}-[-2,0], \beta \neq 0$, because $\mathrm{a}_{\mathrm{ii}} \neq 0$.

Theorem 4: For any matrix that satisfies Eq. 16:

$$
\begin{aligned}
& \operatorname{det}\left(\delta \mathrm{D}-\gamma \mathrm{L}-\gamma^{-1} \mathrm{U}\right)=\operatorname{det}(\delta \mathrm{D}-\mathrm{L}-\mathrm{U}) \\
& \forall \delta, \gamma \in \mathrm{R} \backslash\{0\}
\end{aligned}
$$

In general two cyclic consistently ordered matrix in the sense of Young (1971); Theorem 3.3pag 147) and Varga (1965). The eigenvalues $\beta$ of the KSOR point iteration matrix are related to the eigenvalues $\mu$ of the Jacobi point iteration matrix by the relation Eq. 17:

$$
\left(\beta+\beta \omega^{*}-1\right)^{2}=\beta \omega^{* 2} \mu^{2}, \omega^{*} \neq 0
$$

Proof: The eigenvalues $\beta$ of the KSOR point iteration matrix satisfy:

$$
\begin{aligned}
& \operatorname{det}\left[\left(1-\beta-\beta \omega^{*}\right) D+\omega^{*} \mathrm{U}+\omega^{*} \beta \mathrm{L}\right]=0, \\
& \operatorname{det}\left[\left(\omega^{*} \beta^{\frac{1}{2}}\right)\left\{\frac{\beta+\beta \omega^{*}-1}{\omega^{*} \beta^{\frac{1}{2}}} \mathrm{D}-\beta^{-\frac{1}{2}} \mathrm{U}\right]=0,\right. \\
& \left.-\beta^{\frac{1}{2}} \mathrm{~L}\right\} \\
& \beta \neq 0, \omega^{*} \neq 0 \\
& \operatorname{det}\left[\frac{\beta+\beta \omega^{*}-1}{\omega^{*} \beta^{1 / 2}} \mathrm{D}-\beta^{-1 / 2} \mathrm{U}-\beta^{1 / 2} \mathrm{~L}\right]=0, \\
& \operatorname{det}\left[\frac{\beta+\beta \omega^{*}-1}{\omega^{*} \beta^{1 / 2}} \mathrm{D}-(\mathrm{U}+\mathrm{L})\right]=0 \\
& \operatorname{det}\left[\frac{\beta+\beta \omega^{*}-1}{\omega^{*} \beta^{1 / 2}} \mathrm{I}-\mathrm{D}^{-1}(\mathrm{U}+\mathrm{L})\right]=0
\end{aligned}
$$

Which proves that, $\frac{\beta+\beta \omega^{*}-1}{\omega^{*} \beta^{1 / 2}}=\mu$ is an eigenvalue of the Jacobi iteration matrix. This result gives a direct correspondence between the eigenvalues $\beta$ of the KSOR iteration matrix, $\mathrm{T}_{\mathrm{KSOR}}$ and those of the Jacobi iteration matrix, $T_{j}$. In particular if $T_{j}$ has a p-fold zero eigenvalue, then $\mathrm{T}_{\mathrm{KSOR}}$ has $\mathrm{p}$ corresponding eigenvalues equal to $1 /\left(1+\omega^{*}\right)$.

Moreover, associated with the $2 \mathrm{q}$ nonzero eigenvalues $\pm \mu_{i}$ of ${ }_{j}$ there are $2 q$ eigenvalues of $T_{K S O R}$ which satisfy:

$$
\left(\beta_{\mathrm{q}}+\beta_{\mathrm{q}} \mathrm{w} *-1\right)^{2}=\beta_{\mathrm{q}} \mu_{\mathrm{q}}^{2} \omega^{* 2}
$$

The correspondence between the eigenvalues $\beta$ of the KSOR iteration matrix, $\mathrm{T}_{\mathrm{KSOR}}$ and those $\lambda$ of the
SOR iteration matrix, $\mathrm{T}_{\mathrm{SOR}}$, will be considered in a next work. From the point of view of integration methods for certain systems of linear differential equations, Albrecht and Klein (1984) and the references therein the KSOR method can be considered as the method which uses the prediction correction philosophy in one step. From the point of view of extrapolated methods the KSOR method, like the SOR method can be considered as an extrapolated Gauss Seidel method. The KSOR method and other iterative methods can be combined from the point of view of prediction correction techniques and this will be our considerations in a subsequent work.

The KSOR algorithm: we introduce the algorithmic formulation of the KSOR method. This algorithm is similar except for some constant multipliers of the already well-established SOR algorithm (Burden and Faires, 2005).

\section{Algorithm (KSOR):}

Input: The number of equations m:

- The entries $a_{i j}, 1 \leq i, j \leq m$, of the matrix $A$

- The entries $b_{i}, 1 \leq \mathrm{i} \leq \mathrm{m}$ of $\mathrm{b}$

- The entries $\mathrm{XO}_{\mathrm{i}}, 1 \leq \mathrm{i} \leq \mathrm{m}$ of $\mathrm{XO}=\mathrm{X}^{(0)}$; the parameter $\omega^{*}$

- Tolerance TOL; maximum number of iterations $\mathrm{N}$

Output: The approximate solution $\mathrm{x}_{1}, \ldots \mathrm{x}_{\mathrm{m}}$ or $\mathrm{a}$ message that the number of iterations was exceeded:

Step 1: Set k=1

Step 2: While $(\mathrm{k} \leq \mathrm{N})$ do steps 3-6

Step 3: For $\mathrm{i}=1, \ldots, \mathrm{m}$

$$
\operatorname{setx}_{i}=\frac{1}{\left(1+\omega^{*}\right)} \mathrm{XO}_{\mathrm{i}}+\frac{\omega^{*}}{\mathrm{a}_{\mathrm{ii}}\left(1+\omega^{*}\right)}\left\{\mathrm{b}_{\mathrm{i}}-\sum_{\mathrm{j}=1}^{\mathrm{i}-1} \mathrm{a}_{\mathrm{ij}} \mathrm{x}_{\mathrm{i}}-\sum_{\mathrm{j}=1+1}^{\mathrm{m}} \mathrm{a}_{\mathrm{ij}} \mathrm{XO}_{\mathrm{j}}\right\}
$$

Step 4 : If $\|\mathrm{X}-\mathrm{XO}\|<\mathrm{TOL}$ Then output $\left(\mathrm{x}_{1}, \ldots \mathrm{x}_{\mathrm{n}}\right)$ (Procedure completed successfully.) STOP.

Step 5: Set $\mathrm{k}=\mathrm{k}+1$.

Step 6: For $\mathrm{i}=1, \ldots, \mathrm{m}$ set $\mathrm{XO}_{\mathrm{i}}=\mathrm{xi}$

Step 7: OUTPUT ('Maximum number of iterations exceeded'); (Procedure completed successfully.) STOP

Illustrative examples: Among the test problems we have considered, we mention only two simple well known examples used in the literature with origins in the discretization of boundary value problems (Varga, 1965; Young, 1971). In the first example we present the 
solution values and the graphical representation of the absolute values of the eigenvalues of the SOR and KSOR iteration matrices

In the second example we present the eigenvalues of the Jacobi $\mu_{\mathrm{i}}, \mathrm{I}=1,2,3$ and 4 and Gauss Seidel $v_{\mathrm{i}}, \mathrm{i}=$ $1,2,3$ and 4 iteration matrices and obtained the eigenvalues $\lambda_{\mathrm{i}}, \mathrm{i}=1,2,3$ and 4 of the SOR iteration matrix as functions in $\omega$ and the eigenvalues $\beta_{\mathrm{i}}, \mathrm{i}=1,2,3$ and 4 of the KSOR iteration as functions in $\omega^{*}$.

The main difficulty in the efficient use of iterative methods which depends on some parameters like the SOR method, the AOR method lies in making a good estimate of the optimum relaxation parameters which maximizes the rate of convergence of the method. In the following we consider two well known examples with known optimum relaxation parameter $\omega_{\text {opt }}$. Determining the optimum value of the relaxation parameters is a very important task and it will be considered later in a separt work.

Example 1: Consider a system with data Eq. 18:

$$
A=\left[\begin{array}{cc}
2 & -1 \\
-1 & 2
\end{array}\right], \mathrm{b}\left[\begin{array}{l}
1 \\
1
\end{array}\right]
$$

Whose exact solution is $\mathrm{x}_{1}=1, \mathrm{x}_{2}=1$, (Young page 96) and (Varga, 1965).

It is well known that, for this system we have.

The eigenvalues of the Jacobi iteration matrix $T_{j}$ in this example are:

$\mu_{1}=\mu_{2}=0.5$

The eigenvalues of the Gauss Seidel iteration matrix $\mathrm{T}_{\mathrm{G}}$ are:

$\lambda_{1}=0.0, \lambda_{2}=0.25$ Eq. 19:

The eigenvalues of the SOR iteration matrix $\mathrm{T}_{\mathrm{SOR}}$ are

$$
\lambda_{1,2}=0.125\left\{8-8 \omega+\omega^{2} \pm \omega \sqrt{16-16 \omega+\omega^{2}}\right\}
$$

Figure 1 illustrates the behavior of the absolute values of the eigenvalues of the SOR iteration matrix against the relaxation parameter. The eigenvalues of the KSOR iteration matrix $\mathrm{T}_{\mathrm{KSOR}}$ are Eq. 20:

$$
\beta_{1,2}=\frac{8+8 \omega^{*}+\omega^{* 2} \pm \omega^{*} \sqrt{16+16 \omega^{*}+\omega^{* 2}}}{8\left(1+2 \omega^{*}+\omega^{* 2}\right)}
$$

Table 1:The solution values obtained by the SOR method with $\omega_{\text {opt }}$

\begin{tabular}{|c|c|c|c|}
\hline \multirow{2}{*}{$\mathrm{N}$} & \\
\hline & $\mathrm{x}_{1}^{[\mathrm{n}]}$ & $\mathrm{x}_{2}^{[\mathrm{n}]}$ & $\left|\mathrm{E}_{1}^{\mathrm{KSOR}}\right|$ \\
\hline$\overline{0}$ & 0.000000 & 0.000000 & 0.0000000000 \\
\hline 1 & 0.535000 & 0.821225 & 0.4981820000 \\
\hline 2 & 0.936905 & 0.978759 & 0.0665742000 \\
\hline 3 & 0.993052 & 0.997770 & 0.0072966400 \\
\hline 4 & 0.999293 & 0.999778 & 0.0007407840 \\
\hline 5 & 0.999931 & 0.999978 & 0.0000725687 \\
\hline 6 & 0.999993 & 0.999998 & $\downarrow$ \\
\hline 7 & 0.999999 & 1.000000 & \\
\hline 8 & 1.000000 & 1.000000 & \\
\hline
\end{tabular}
SOR

$\omega_{\text {opt }}=1.07 \rho=0.0942179$

Table 2: The solution values obtained by the KSOR method KSOR

$\omega^{*}=-13.513 \rho=0.0799169$

\begin{tabular}{llll}
$\mathrm{N}$ & $\mathrm{x}_{1}^{[\mathrm{n}]}$ & $\mathrm{x}_{2}^{[\mathrm{n}]}$ & $\left|\mathrm{E}_{1}^{\mathrm{KSOR}}\right|$ \\
\hline 1 & 0 & 0 & 0.00000000000 \\
2 & 0.539958 & 0.831514 & 0.48992400000 \\
3 & 0.945789 & 0.984193 & 0.05646800000 \\
4 & 0.995797 & 0.998994 & 0.00432127000 \\
5 & 0.999793 & 0.999968 & 0.00020972500 \\
6 & 1.000000 & 1.000000 & 0.00000231632 \\
7 & 1.000000 & 1.000000 & $\downarrow$ \\
8 & 1.000000 & 1.000000 & \\
\hline
\end{tabular}

Table 3: The solution values obtained by the KSOR method KSOR

$\omega^{*}=-14.9282 \rho=0.07179687$

\begin{tabular}{clll}
$\mathrm{N}$ & $\mathrm{x}_{1}^{[\mathrm{n}]}$ & $\mathrm{x}_{1}^{[\mathrm{n}]}$ & $\left|\mathrm{E}_{1}^{\text {KSOR }}\right|$ \\
\hline 1 & 0.535898 & 0.823085 & 0.4966780 \\
2 & 0.938513 & 0.979751 & 0.0647356 \\
3 & 0.993563 & 0.998004 & 0.0067391 \\
4 & 0.999393 & 0.999818 & 0.0006341 \\
5 & 0.999946 & 0.999984 & $5.631 \mathrm{E}-05$ \\
6 & 0.999995 & 0.999999 & $4.818 \mathrm{E}-06$ \\
7 & 1.000000 & 1.000000 & $4.015 \mathrm{E}-07$ \\
8 & & & $\downarrow$ \\
\hline
\end{tabular}

By simple calculations one can easily see that, theorem 4, formula (17) is completely satisfied since every $2 \times 2$ matrix is a consistently ordered matrix. Table 1-3 illustrate the component wise solution, where $E_{1}$ is the Euclidian norm of the error defined by the relation:

$$
\left|E_{1}^{S O R}\right|=\sqrt{\sum_{\mathrm{i}=1}^{\mathrm{m}}\left(\mathrm{x}_{\text {exact }}-\mathrm{x}_{\mathrm{i}}^{[\mathrm{n}]}\right)^{2}}
$$

Figure 2 illustrates the behavior of the absolute value of the eigenvalues of the KSOR iteration matrix $\mathrm{T}_{\mathrm{KSOR}}$ agains the relaxation parameter.

Example 2: Consider a system with Eq. 21: 


$$
A=\left[\begin{array}{cccc}
4 & -1 & -1 & 0 \\
-1 & 4 & 0 & -1 \\
-1 & 0 & 4 & -1 \\
0 & -1 & -1 & 4
\end{array}\right], b=\left[\begin{array}{l}
2 \\
2 \\
2 \\
2
\end{array}\right]
$$

For simplicity we adapted the right hand side $\mathrm{b}$ so that the exact solution is $\mathrm{x}_{1}=1, \mathrm{x}_{2}=1 \mathrm{x}_{3}=1 \mathrm{x}_{4}=1$, (Young, 1971; Varga, 1965).

It is well known that, for this system we have.

The eigenvalues of the Jacobi iteration matrix $T_{j}$ are the roots of the equation:

$$
\begin{aligned}
& \mu^{4}-0.25 \mu^{2}=0 \\
& \mu_{1}=\mu_{2}=0, \mu_{3}=-0.5, \mu_{4}=0.5
\end{aligned}
$$

The eigenvalues of the Gauss Seidel iteration matrix $\mathrm{T}_{\mathrm{G}}$ are the roots of the equation:

$$
\begin{aligned}
& v^{4}-0.25^{3} v^{3}=0 \\
& v_{1}=v_{2}=v_{3}=0.0, v_{4}=0.25
\end{aligned}
$$

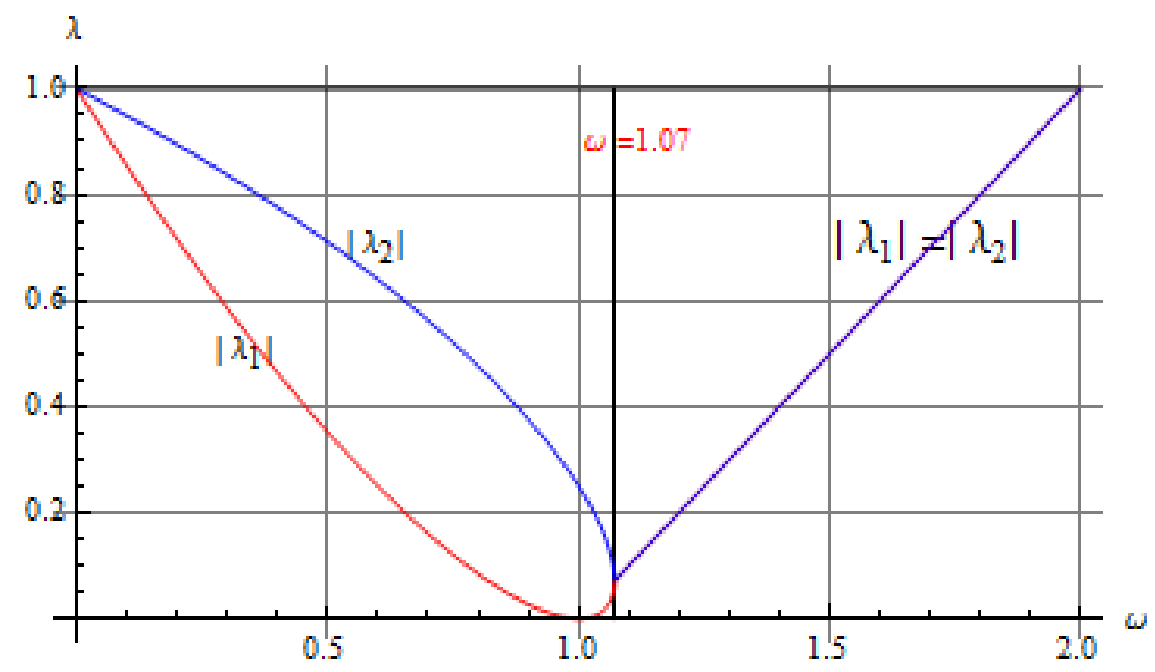

Fig. 1: The behavior of the absolue value of the eigenvalues of iteration matrix $\mathrm{T}_{\mathrm{SOR}}$

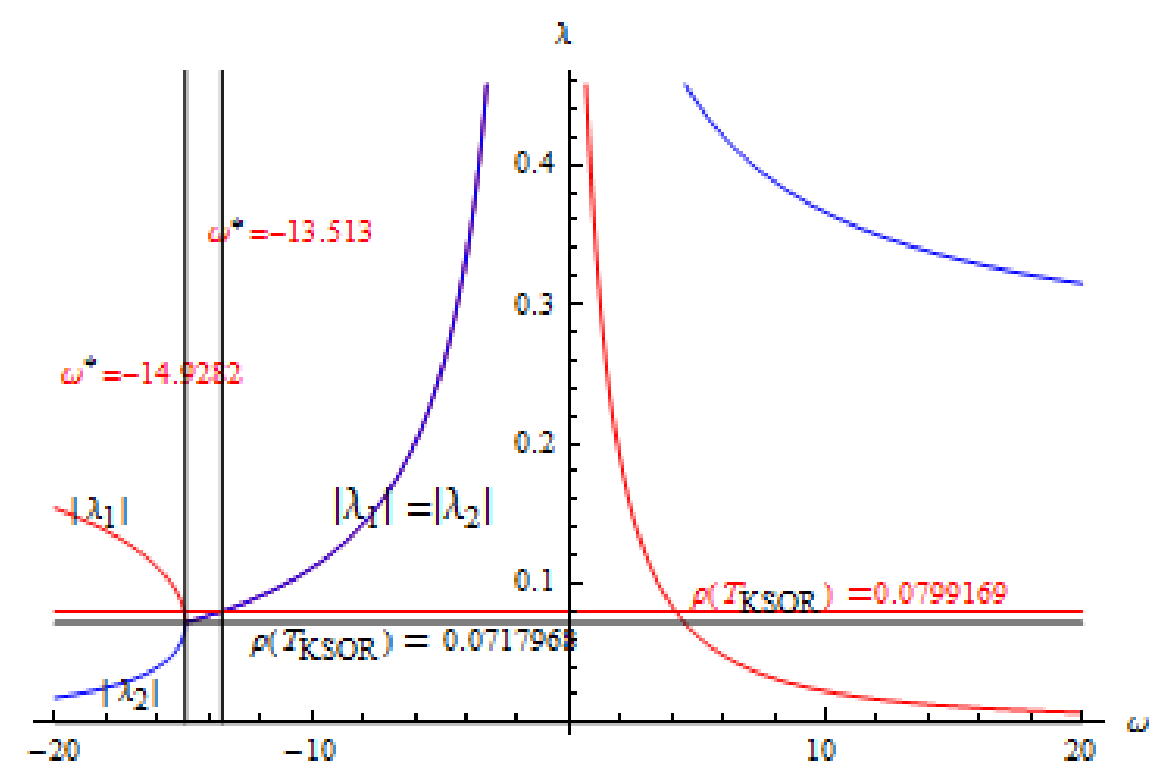

Fig. 2: The behavior of the absolute values of the eigenvalues of the iteration matrix $\mathrm{T}_{\mathrm{KSOR}}$ 


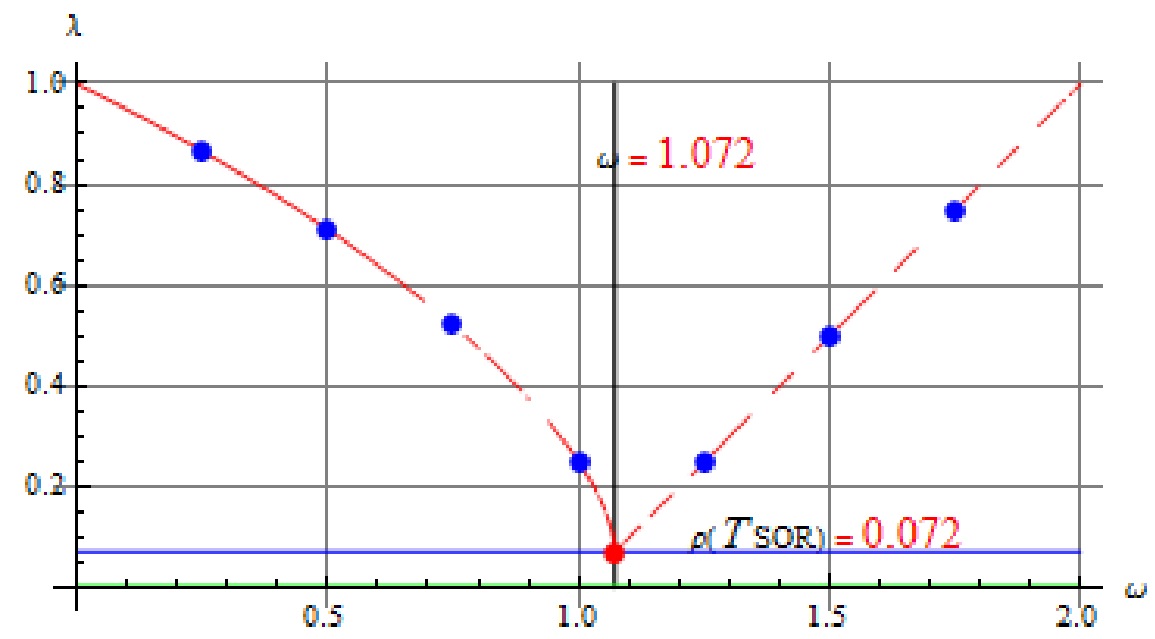

Fig. 3: The behavior of the spectral radius of the iteration matrix $\mathrm{T}_{\mathrm{SOR}}$

Table 4: The eigen values of the $\mathrm{T}_{\mathrm{SOR}}$ corresponding to those of $\mathrm{T}_{\mathrm{J}}$

\begin{tabular}{llllll}
\hline$\omega$ & $\lambda_{1}$ & $\lambda_{2}$ & $\lambda_{3}$ & $\lambda_{4}$ & $\max \left|\lambda_{i}\right|$ \\
\hline 0.250 & 0.750 & 0.750 & 0.649278 & 0.866347 & 0.866347 \\
0.500 & 0.500 & 0.500 & 0.351732 & 0.710768 & 0.710768 \\
0.750 & 0.250 & 0.250 & 0.120062 & 0.520563 & 0.520563 \\
1.000 & 0.000 & 0.000 & 0.000000 & 0.250000 & 0.250000 \\
1.072 & -0.072 & -0.072 & $0.071648 \pm 0.00711084 \mathrm{I}$ & & 0.072000 \\
1.250 & -0.250 & -0.250 & $-0.0546875 \pm 0.243945 \mathrm{I}$ & & 0.250000 \\
1.500 & -0.500 & -0.500 & $-0.21875 \pm 0.446909 \mathrm{I}$ & & 0.500000 \\
1.750 & -0.750 & -0.750 & $-0.367188 \pm 0.653967 \mathrm{I}$ & & 0.750000 \\
\hline
\end{tabular}

Table 5:The behavior of the spectral radius of the $\mathrm{T}_{\mathrm{SOR}}$ near the minimum value

\begin{tabular}{lc}
\hline$\omega$ & $\rho\left(\mathrm{T}_{\mathrm{SOR}}\right)$ \\
\hline 1.070 & 0.0942179 \\
1.071 & 0.0864472 \\
1.072 & 0.0720000 \\
1.073 & 0.0730001 \\
1.074 & 0.0740000 \\
1.075 & 0.0750000 \\
\hline
\end{tabular}

The SOR iteration matrix $\mathrm{T}_{\mathrm{SOR}}$ is Eq. 22:

$$
\mathrm{T}_{\mathrm{SOR}}=\left[\begin{array}{cccc}
(1-\omega) & 0.25 \omega & 0.25 \omega & 0 \\
0.25(1-\omega) & \mathrm{t}_{2} & 0.625 \omega^{2} & 0.25 \omega \\
0.25 \omega(1-\omega) & 0.625 \omega^{2} & \mathrm{t}_{2} & 0.25 \omega \\
0.125 \omega^{2}(1-\omega) & \mathrm{t}_{3} & \mathrm{t}_{3} & \mathrm{t}_{1}
\end{array}\right]
$$

Where:

$$
\begin{aligned}
& \mathrm{t}_{1}=(1-\omega)+0.125 \omega^{2} \\
& \mathrm{t}_{2}=(1-\omega)+0.625 \omega^{2} \\
& \mathrm{t}_{3}=0.25 \omega(1-\omega)+003125 \omega^{3}
\end{aligned}
$$

In Table 4 we list the calculated numerical values of the eigenvalues of the iteration matrix defined in (22) and the maximum absolute value (the spectral radius) as shown in Fig. 3.

The KSOR iteration matrix $\mathrm{T}_{\mathrm{KSOR}}$ is Eq. 23:

$$
\mathrm{T}_{\mathrm{KSOR}}=\frac{1}{\sigma}\left[\begin{array}{ccrc}
4 \sigma_{1} & \omega^{*} \sigma_{1} & \omega^{*} \sigma_{1} & 0 \\
4 \sigma_{2} & \omega^{*} \sigma_{2}+4 \sigma_{1} & \omega^{*} \sigma_{2} & \omega^{*} \sigma_{1} \\
4 \sigma_{2} & \omega^{*} \sigma_{2} & \omega^{*} \sigma_{2}+4 \sigma_{1} & \omega^{*} \sigma_{1} \\
\sigma_{4} & \omega^{*} \sigma_{3}+4 \sigma_{2} & \omega^{*} \sigma_{3}+4 \sigma_{2} & 2 \omega^{*} \sigma_{2}+4 \sigma_{1}
\end{array}\right]
$$

Where:

$$
\begin{aligned}
& \sigma=256+1024 \omega^{*}+1536 \omega^{* 2}+1024 \omega^{* 3}+256 \omega^{* 4} \\
& \sigma_{1}=64+192 \omega^{*}+192 \omega^{* 2}+64 \omega^{* 3} \\
& \sigma_{2}=16 \omega^{*} 32 \omega^{* 2}+16 \omega^{* 3} \\
& \sigma_{3}=8 \omega^{* 2}+8 \omega^{* 3}
\end{aligned}
$$

In Table 6 we list the calculated numerical values of the eigenvalues of the iteration matrix defined in (23) and the maximum absolute value (the spectral radius) as shown in Fig. 4.

It is clear from, Table 4 that corresponding to $\mu_{\mathrm{i}}=$ $0, i=1,2 \lambda_{i}=1-\omega, i=1,2$ and the relation between the eigenvalues $\mu_{\mathrm{i}}, \lambda_{\mathrm{i}}$ and the relaxation parameter $\omega$, $\mu_{\mathrm{i}} \omega \lambda_{\mathrm{i}}^{1 / 2}=\lambda_{\mathrm{i}}+\omega-1$ is completely satisfied.

It is clear from, Table 6 , that corresponding to $\mu_{\mathrm{i}}=$ $0, \mathrm{i}=1,2, \beta_{\mathrm{i}}=\frac{1}{1+\omega^{*}}, \mathrm{i}=1,2$ and the relation, between the eigenvalues $\mu_{\mathrm{i}}, \beta_{\mathrm{i}}$ and the relaxation parameter $\omega^{*}$, theorem(4), $\mu_{\mathrm{i}} \omega^{*} \beta_{\mathrm{i}}{ }^{1 / 2}=\beta_{\mathrm{i}}+\beta_{\mathrm{i}} \quad \omega^{*}-1$ is completely satisfied all calculations and graphs are performed with the help of the computer algebra system Mathematica 7.0. 


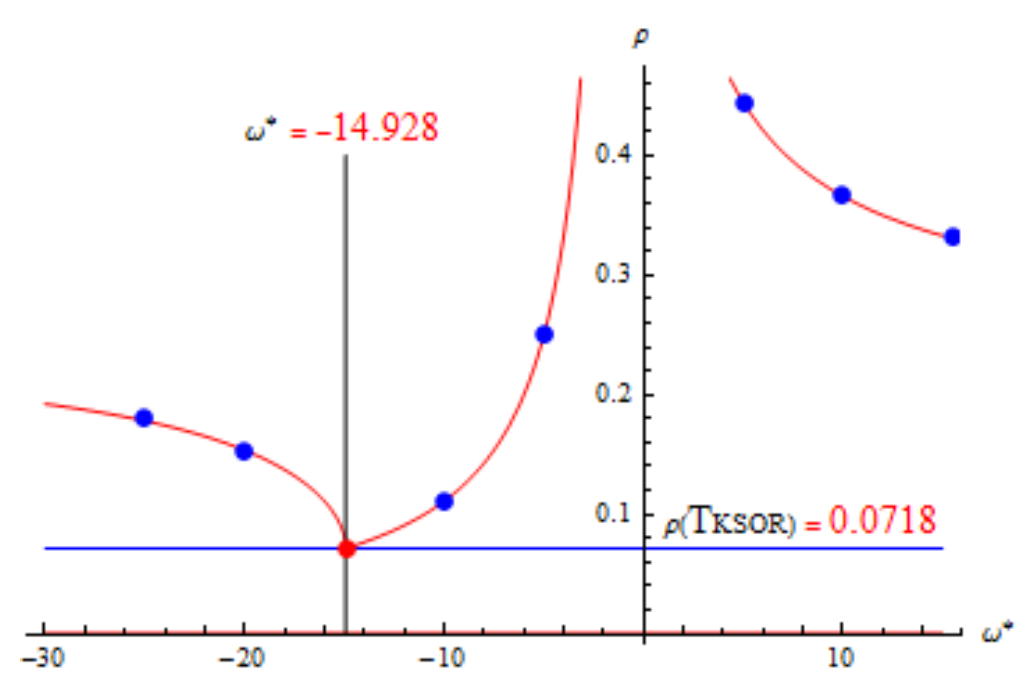

Fig. 4: The behavior of the spectral radius of the iteration matrix $T_{K S O R}$

Table 6: The eigen values of the $T_{\text {KSOR }}$ corresponding to those of $T_{J}$

\begin{tabular}{lcclcl}
\hline$\omega^{*}$ & $\beta_{1}$ & $\beta_{2}$ & \multicolumn{1}{c}{$\beta_{3}$} & $\beta_{4}$ & $\max \left|\beta_{\mathrm{i}}\right|$ \\
\hline-50.0 & -0.0204082 & -0.0204082 & 0.00191423 & 0.217578 & 0.217578 \\
-25.0 & -0.0416670 & -0.0416670 & 0.00974298 & 0.178191 & 0.178191 \\
-20.0 & -0.0526316 & -0.0526316 & 0.01801970 & 0.153725 & 0.153725 \\
-14.928 & -0.0717980 & -0.0717980 & $0.071796 \pm 0.0005104 \mathrm{I}$ & & 0.071798 \\
-10.0 & -0.1111110 & -0.1111110 & $0.0432099 \pm 0.102365 \mathrm{I}$ & & 0.111111 \\
-5.0 & -0.2500000 & -0.2500000 & $0.0546875 \pm 0.243945 \mathrm{I}$ & & 0.25000 \\
1.0 & 0.5000000 & 0.5000000 & 0.3517320 & 0.710768 & 0.710768 \\
5.0 & 0.1666670 & 0.1666670 & 0.062500 & 0.444444 & 0.444444 \\
10.0 & 0.0909091 & 0.0909091 & 0.0225904 & 0.365839 & 0.365839 \\
15.0 & 0.0625000 & 0.0625000 & 0.0117306 & 0.332996 & 0.332996 \\
\hline
\end{tabular}

Table 7:The behavior of the spectral radius of the $\mathrm{T}_{\mathrm{KSOR}}$ near the minimum value

\begin{tabular}{ll}
\hline$\omega^{*}$ & $\rho\left(\mathrm{T}_{\text {KSOR }}\right)$ \\
\hline-14.925 & 0.0718133 \\
-14.926 & 0.0718081 \\
-14.927 & 0.0718030 \\
-14.928 & 0.0717978 \\
-14.929 & 0.0728104 \\
-14.930 & 0.0733212 \\
\hline
\end{tabular}

\section{RESULTS}

- The KSOR updates the residue simultaneously with the solution in addition to the use of the most recent calculated solution which reflects the the rapied convergence at the begainning appeared in the numerical examples

- The domain of the relaxation parameter in the KSOR is $\omega^{*} \in \mathrm{R}-[-2,0]$ instead of $\omega \in(2,0)$ in the SOR

- The iteration matrix of the proposed method, the consistency and convergence analysis of the method are well established

- Afunctional eigenvalue relation between the eigenvalues of the iteration matrices and the relaxation parameters (theorem (4)) is well established
- Numerical examples illustrating and confirming the theoritical eigenvalue functional relation are considered

- From Table 5-7, we see that the spectral radius $\rho\left(T_{\text {SOR }}\right)$ changes from $0.072-0.092$ while $\rho\left(T_{K S O R}\right)$ changes from 0.072-0.073 in an interval of length 0.005 around the minimum value i.e., the change in $\rho\left(T_{\mathrm{SOR}}\right)$ is 20 times the change in $\rho\left(\mathrm{T}_{\mathrm{KSOR}}\right)$ along an interval of the same length which illustrates relaxation of the sensitivity around the minimum value

- $\quad$ Further extensions are mentioned

\section{DISCUSSION}

Although the problem of solving large sparse linear systems of algebraic equations is one of the old problems (Saad and Vorst, 2000; Hackbusch, 1994) it is still has an important role in many modern areas of science. The SOR is one of most used iterative methods espcially when a good estimation of the optimum value of the relaxation parameter $\omega_{\text {opt }}$ is avaliable. Even if $\omega_{\text {opt }}$ is avaliable, it is sensitive as illustrated in the results of the numerical examples.

In comparison with the SOR method, with known optimal value of the relaxation parameter, the KSOR method has the same advantages of the SOR. Even from the point of view of the splitting of the coefficient matrix, one can see that the SOR uses the splitting $\mathrm{A}=\frac{1}{\omega}((\mathrm{D}-\omega \mathrm{L})-((1-\omega) \mathrm{D}+\omega \mathrm{U}))$ while in the KSOR it is $\mathrm{A}=\frac{1}{\omega^{*}}\left(\left(\left(1+\omega^{*}\right) \mathrm{D}-\omega^{*} \mathrm{~L}\right)-\left(\mathrm{D}+\omega^{*} \mathrm{U}\right)\right)$ in addition to the 
possibility of the use of the philosophy of the prediction correction techniques, which we will consider in a subsequent work.

It remains to introduce an effective procedure for the estimation of the optimum value of the relaxation parameter $\omega^{*}$ opt. which maximizes the rate of convergence of the proposed KSOR method and this will be the objective of a subsequent work. Also the KSOR can be used with more relaxation parameters as well as combinations of the SOR and the KSOR can be considered.

\section{CONCLUSION}

The KSOR has the same simple structure as the SOR method so its implementation is an easy task. The theoretical properties, the convergence as well as the consistency of the proposed method are proved. Comparison with other iterative methods especially with the SOR, with known optimal value of the relaxation parameter is discussed.

From the the computational point of view the method has the advantage of updating the first component from the first step unlike the other iterative methods which reflects the rapid convergence at the beginning.

The study of the spectral radius of the iteration matrices, which is a measure of the convergence rate of the linear iterative methods, have proved that there is a value of the relaxation parameter $\omega^{*}$ for which $\rho\left(\mathrm{T}_{\mathrm{KSOR}}\right)$ is comparable with that of the SOR corresponding to $\omega_{\text {opt }}$. The numerical examples have confirmed the theoretical eigenvalue functional relation (theorem 4) and illustrated that the extension of the domain of the relaxation parameter has the the effect of relaxing the sensitivity $\rho\left(\mathrm{T}_{\mathrm{SOR}}\right)$ around its minimum value.

\section{REFERENCES}

Albrecht, P. and M.P. Klein, 1984. Extrapolated iterative methods for linear systems. SIAM. J. Numer. Anal., 21: 192-201.

Avdelas, G. and A. Hadjidimos, 1981. Optimum accelerated overrelaxation method in a special case. Math. Comput., 36: 183-187.
Burden, R.L. and J.D. Faires, 2005. Numerical Analysis. 8th Edn., Thomson Brooks/Cole, Pacific Grove, Calif., ISBN-10: 0534404995, pp: 847.

Darvishi, M.T. and P. Hessari, 2006. On convergence of the symmetric modified successive overrelaxation method for 2-cyclic coefficient matrices. Applied Math. Comput., 183: 953-960. DOI: 10.1016/j.amc.2006.05.121

Darvishi, M.T. and P. Hessari, 2011. A modified symmetric successive overrelaxation method for augmented systems. Comput. Math. Appli., 61: 3128-3135. DOI: 10.1016/j.camwa.2011.03.103

Hackbusch, W., 1994. Iterative Solution of LARGE Sparse Systems of Equations. 1st Edn., SpringerVerlag, New York, ISBN-10: 0387940642, pp: 429.

Hadjidimos, A., 1978. Accelerated overrelaxation method. Math. Comput., 32: 149-157.

Louka, M.A., N.M. Missirlis and F.I. Tzaferis, 2009. Is modified PSD equivalent to modified SOR for twocyclic matrices? Linear Algebra Appli., 432: 27982815. DOI: 10.1016/j.laa.2009.12.016

Papadomanolaki, M.G., E.P. Papadopoulou and Y.G. Saridakis, 2010. p-Cyclic SOR for BVPs with periodic boundary conditions. Applied Numerical Math., 60: 411-419. DOI: 10.1016/j.apnum.2009.08.006

Saad, Y. and H.A.VD. Vorst, 2000. Iterative solution of linear systems in the 20th century. J. Comput. Applied Math., 123: 1-33. DOI: 10.1016/S03770427(00)00412-X

Salkuyeh, D.K. and F. Toutounian, 2006. Numerical accuracy of a certain class of iterative methods for solving linear system. Applied Math. Comput., 176: 727-738. DOI: 10.1016/j.amc.2005.10.019

Varga, R.S., 1965. Matrix Iterative Analysis. 3rd Edn., Prentice-Hall, Englewood Cliffs, NJ., pp: 322.

Wang, C.L., 2010. Convergences of splitting iterative methods for symmetric indefinite linear systems, Applied Math. Comput., 216: 1687-1693. DOI: 10.1016/j.amc.2009.12.035

Young, D., 1954. Iterative methods for solving partial difference equations of elliptic type. Trans. Am. Math. Soc., 76: 92-111.

Young, D.M., 1971. Iterative Solution of Large Linear Systems. 1st Edn., Academic Press, New York, pp: 570 . 